\title{
Dos inscripciones funerarias ${ }^{1}$
}

\section{Two burial inscriptions}

\author{
Salvador Ordóñez Agulla \\ Universidad de Sevilla
}

\section{RESUMEN}

Se ofrece la edición completa de dos inscripciones funerarias procedentes del sur de la Península Ibérica, una de ellas de texto ya conocido (Hep 13, 260), procede de Chipiona (Cádiz); la otra, aún inédita, es de procedencia desconocida y se conserva en una colección particular.

\section{SUMMARY}

The publication of two funerary inscriptions from the South of the Iberian Peninsula is here in offered. One of them comes from Chipiona (Cádiz), and its text is already known (Hep 13, 260); the other one, yet unpublished, is of unknown origin and belongs to a private collection.

PALABRAS CLAVES: epígrafe romano, epitafio, Hispania.

KEY WORDS: roman epigraph, epitaph, Hispania.

\section{EPITAFIO DE AEMILIA GYMNAS}

La ciudad de Chipiona, donde se ubicaba el antiguo

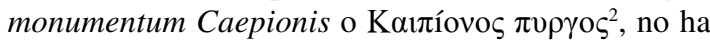
sido demasiado pródiga en la transmisión de informaciones sobre su pasado romano. A los hallazgos aislados acaecidos desde el siglo XVII, especialmente los registrados por Diego de Carmona y Bohórquez en su historia del Monasterio de Regla ${ }^{3}$, se suman

1 Trabajo realizado en el marco del proyecto $\mathrm{I}+\mathrm{D}+\mathrm{i}$ HAR2009-02283, del MICINN. Hemos de agradecer las observaciones críticas y sugerencias efectuadas por los revisores anónimos del trabajo. Obviamente, la responsabilidad del texto es exclusiva del autor

${ }^{2}$ Mela, Chor. 3.4; Str. 3.1.9; Iord. Get. 44.230: Scipionis monumentum.

${ }^{3}$ Diego de Carmona y Bohórquez, Historia sacra del insigne origen y raro aparecimiento de la antiquisima imagen de Nuestra Señora de Regla y de sus admirables y maravillosas obras, obra inédita que hemos podido consultar gracias a los buenos oficios del Dr. A. Ramos Millán. Fue colacionada en Muñoz y Romero 1858: 222. desde hace unos años los resultados de la investigación arqueológica desarrollada desde los años 80 del pasado siglo. Esta labor reciente ha puesto de relieve la intensidad del poblamiento rural en el término municipal con la detección de un conjunto de asentamientos rurales, algunos de los cuales presentan una específica vocación hacia la producción alfarera relacionada con las industrias vinaria y de salazones, de forma similar a otros lugares del entorno de la Bahía gaditana en época altoimperial (Ramos 1981; Ramos y Riesco 1983; Alarcón 1991; Alcázar et alii 1994; Lagóstena 2001; Ramos Millán et alii 2001; Fornell 2005: 101-102, 108).

Y tampoco son numerosas las inscripciones originarias del lugar. Solo cuatro textos se han conservado en los aproximadamente 800 años en los que la práctica epigráfica se estuvo ejerciendo en el sur peninsular. Dos son de época tardoantigua, conocidos por la tradición manuscrita y hoy en paradero desconocido, procedentes del recinto del monasterio de Regla, en un entorno de "infinitos sepulcros, piedras y huesos que se descubren de ordinario en aquel sitio" ". Se trata de los epitafios de un ignoto famulus Dei de seis años que muere en $466^{5}$, el otro, el de una virgo llamada $U_{r b a n a^{6}}$, fechado por Hübner en el siglo VII d.C. ${ }^{7}$.

\footnotetext{
${ }^{4}$ Ambas inscripciones se recogen, respectivamente, en el capítulo X (ff. 55 ss.) y XV (ff. 78 ss.) de la obra de Carmona y Bohórquez. En f. 80a-r recoge el testimonio del reciente hallazgo de otras sepulturas desprovistas de tituli, así como de una de ellas que recordaba la presencia de un mártir, pero cuyo tenor no pudo transmitir por pérdida de las notas tomadas sobre esta pieza.

${ }^{5}$ IHC $113=$ ICERV $141=$ Diehl 1424D adn.

${ }^{6}$ IHC $112=$ ICERV 147.

${ }^{7}$ Este epitafio fue atribuido por Hübner al siglo VII d.C., mientras que por su parte Vives prefería asignarlo a época postvisigótica, aunque a pesar de ello lo mantuvo en su corpus; pero hay que estar de acuerdo con Muñoz 1995: 180 en que la existencia de paralelos paleocristianos de la fórmula requiescit in domino en otras regiones del imperio permite considerar válida la cronología del sabio alemán.
} 
Las otras dos piezas, de cronología imperial, son también funerarias. Una de ellas corresponde al epitafio de C. Canius Primulus, que se fecha a finales del siglo II d.C., hoy depositado en el museo habilitado en el Santuario de Regla ${ }^{8}$. Pero lo que nos interesa ahora es ofrecer la edición completa de una pieza de la que hasta el momento solo se disponía de una noticia escueta que se dio a conocer en el Anuario Arqueológico de Andalucía de 2001, publicado en 2004 (Ramos et alii 2001: 58), con la sola lectura y una propuesta de datación realizada por nosotros, a petición de los autores, sobre la base de una fotografía. Posteriormente Hispania Epigraphica se hizo eco de ella en la entrada correspondiente del volumen $13^{9}$. Gracias a la amabilidad del Dr. Antonio Ramos Millán pudimos acceder en 2007 a la pieza y efectuar su autopsia, en el marco de un ciclo de conferencias sobre la Antigüedad de Chipiona celebrado en septiembre de ese mismo año. Resultado de aquella autopsia es el breve estudio que se ofrece a continuación.

La inscripción fue hallada en la excavación de urgencia realizada en el yacimiento denominado "El Olivar", en 2001, situado a unos dos km de la línea costera, villa dotada de un complejo de producción alfarera de envases de vino y salazones y salsas de pescado, con sus hornos correspondientes, cuya actividad se prolonga desde época republicana hasta el Bajo Imperio. Concretamente la pieza fue encontrada en un depósito doméstico de basuras sito sobre los restos del derrumbe de la cúpula de la cámara de cocción de uno de los hornos, el $\mathrm{n}^{\circ} 2$, que colmataba los socavones originados por la caída de la mencionada bóveda sobre la parrilla (Ramos et alii 2001: 58).

Se trata de una placa rectangular de mármol blanco de grano grueso, fracturada de antiguo en dos trozos que fueron hallados en dos sectores diferentes de la excavación (sectores 3 y 4). Los márgenes inferior e izquierdo están perfectamente cortados, mientras que el superior y el derecho presentan un desbastado más grosero, lo que permite pensar en una reutilización de una pieza de mayor tamaño que fue adaptada para un nuevo uso. Tanto la parte anterior como la posterior fueron pulimentadas, si bien hoy presentan numerosas concreciones en su superficie, que no dificultan, en cualquier caso, la lectura. Aunque el estado de conservación es bueno, puede observarse claramente que ambos fragmentos han experimentado una diferente meteorización, resultado de la mencionada deposición

\footnotetext{
${ }^{8}$ González 1982: 57 n $82=A E$ 1982, $557=$ HEp on line 1049: C(aius) Canius / Primulus / an(norum) XXXV / h(ic) $s($ itus) e(st) s(it) t(ibi) t(erra) l(evis).

${ }^{9}$ HEp 13, 2003-2004 (2007) 260; HEp on line 25163.
}

en dos lugares diferentes. Se aprecia igualmente en la parte posterior inferior una rebaba de anchura desigual, producto del corte de la sierra y posterior fragmentación para separar la pieza del bloque. Sus medidas son las siguientes: 24 x $(35-33,5)$ x 2,9 cm. Las letras son capitales en 1. 1, con algún rasgo librario en los trazos ascendentes de M; en el resto de líneas es una libraria muy estilizada, con refuerzos poco marcados, con excepción de algunos caracteres ( $\mathrm{S}$ en 1. 1), e incisión poco profunda en todos los caracteres excepto en la 1 . $1 .^{10}$ El módulo de las letras es variable: $3,5-3 \mathrm{~cm}$ en 1. $1,3 \mathrm{~cm}$ en $1.2,2,5-3 \mathrm{~cm}$ en $1.3,2,8-4 \mathrm{~cm}$ en 1.4 . Los espacios interlineales son de $1,5 \mathrm{~cm}$ entre todas las líneas. La ordinatio es aparentemente cuidada y con justificación centrada, aunque se aprecian algunas inconsistencias como la altura diferencial de las letras D y $\mathrm{S}(3,5 \mathrm{~cm})$ con respecto a $\mathrm{M}(3 \mathrm{~cm})$ en 1. 1. Puesto que el margen izquierdo ha sido recortado, la distancia del texto al borde izquierdo en su estado original debía ser la misma aproximadamente que la del lado derecho, esto es, 1. 1: $10,5 \mathrm{~cm} ; 1.2: 8 \mathrm{~cm}$; 1. 3: $13,5 \mathrm{~cm} ; 1.4: 9 \mathrm{~cm}$. El cuadratario no parece haberse guiado por la línea de caja, de la que no se conservan restos, pues se observa cómo los caracteres, especialmente en 1.2 y 4 , no se alinean con respecto a ella. La interpunción en 1.1 entre D y M es un trazo casi horizontal, mientras que entre $\mathrm{M}$ y $\mathrm{S}$, y tras este carácter, se han empleado trazos oblicuos similares a apices. En el resto de las líneas las interpunciones son triangulares con el vértice hacia abajo. Los trazos horizontales de algunas letras, como H, E, A, están inclinados hacia la izquierda, aunque en 1.3 no se ha grabado el de A; el trazo horizontal de L, muy corto, sobresale por la izquierda en 1. 2; H proyecta su trazo vertical izquierdo por encima de la caja de la letra, al igual que $\mathrm{Y}$ lo hace con el trazo diagonal derecho y $\mathrm{X}$ con su diagonal superior derecha (Fig. 1).

El texto dice:

$\mathrm{D} \cdot \mathrm{M} \cdot \mathrm{S} \cdot$

AEMILIA $\cdot$ GYMNAS

ANN $\cdot$ XVII

$\mathrm{H} \cdot \mathrm{S} \cdot \mathrm{E} \cdot \mathrm{S} \cdot \mathrm{T} \cdot \mathrm{T} \cdot \mathrm{L}$

$D($ is) M(anibus) s(acrum) / Aemilia Gymnas / ann(orum) XVII / h(ic) s(ita) e(st) s(it) t(ibi) t(erra) l(evis)

\footnotetext{
${ }^{10}$ Esta combinación de capitales en la dedicación a los Manes y resto del texto en libraria se encuentra también en epígrafes procedentes del valle del Guadalquivir, como CILA II.1, 187 y 190-191, de Celti, o CILA II.1, 312 y 315, de Ilipa: también en las campiñas, $C I L \mathrm{II}^{2} / 5,102$ (Tucci), $C I L \mathrm{II}^{2} / 5,353$ (Monte Horquera).
} 


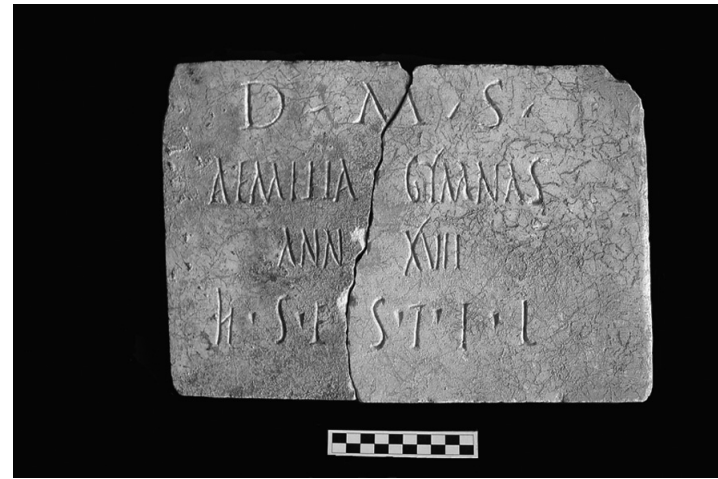

Figura. 1. Inscripción de Aemilia Gymnas (Foto: S. Ordóñez Agulla).

Se trata de una inscripción funeraria del tipo más usual en la provincia Baetica (Stylow 2002: 353), y particularmente en la zona gaditana, con mención del nombre de la difunta sin filiación, la edad y la clásica fórmula funeraria final ${ }^{11}$. El nombre de la difunta se ha hecho constar en nominativo, como es lo usual en la Bética en las fechas en que se redacta el texto. El nomen Aemilius, bastante frecuente en la Península Ibérica (Abascal 1994: 72), está suficientemente atestiguado en el entorno de la bahía gaditana, encontrándose individuos que portan ese gentilicio en diferentes puntos de la misma, como Gades (González 1982: 95-96 no 147-149; González 1982: 200 n $376=H E p$ 6, 526, 380), Chiclana (González 1982: 94 no 146), Baelo (González 1982: 39 n 54 = Bonneville et alii 1988: no 22), Carissa ${ }^{12}$ y Ceret (González 1982: 77 $\mathrm{n}^{\circ} 117=A E$ 1982, 559). El cognomen Gymnas es de filiación griega (Solin 2003: 930) ${ }^{13}$, circunstancia que unida a la ausencia de filiación permite pensar en una joven difunta con la condición de liberta. El contenido etimológico del nombre hace alusión a los ejercicios corporales y al mundo del gymnasion, como otros de la misma familia -Gymnasio(n), Gymnasius-a, Gymnicus-. Pero si todos ellos están bien representados en el mundo romano ${ }^{14}$, no ocurre lo mismo con Gymnas. En la Península Itálica solo se ha documentado por el momento en Roma y en Pola, en los epitafios de una esclava de mediados del siglo II d.C. ${ }^{15}$ y en la inscripción funeraria de un liberto imperial dedicada por sus hijas Armonia y Gymnas ${ }^{16}$. Por su parte, en el

\footnotetext{
${ }^{11}$ Carece, no obstante, de uno de los rasgos característicos de los epígrafes funerarios del área gaditana, la fórmula cara in suis; vide sobre ella Gimeno y Stylow 2003: 201-202.

${ }^{12}$ HEp 11, 146.

${ }^{13}$ No obstante, no se encuentra recogido en Pape y Benseler 1959.

${ }^{14}$ Vide ejemplos en Solin 1989: 101-102.

${ }^{15} C I L$ VI $10018=A E$ 1967, $32=$ Ferrua 1966: $20 \mathrm{n}^{\circ} 4$; vide Solin 1996: 450.

${ }^{16}$ CIL V $41=$ ILS $1644=$ InscrIt 10.1, 50.
}

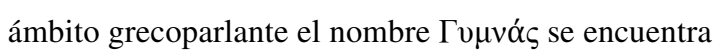
atestiguado en Isauria ${ }^{17}$, mientras que su correspondiente masculino, Gymnus, no representado por el momento en la antroponimia latina, lo está en griego en Egipto, en un texto de tipo proskynema procedente de Silsilis ${ }^{18}$. Con la pieza de Chipiona, pues, tenemos el primer testimonio en la Península Ibérica de este poco común nombre ${ }^{19}$. La ordinatio de la inscripción, con el extenso espacio existente hasta el margen de la inscripción, permite descartar la posibilidad de que se hubiese escrito abreviadamente el nombre Gimnasio, cognomen femenino bien documentado en la propia $U r b s^{20}$.

A juzgar por la consignación de la consagración a los Dioses Manes, la indicación de la edad en ann. y las fórmulas finales (Stylow 1995: 222-223), así como por la paleografía de las letras, esta inscripción debe fecharse en las últimas décadas del siglo II d.C. y primeras del siglo III.

\section{INSCRIPCIÓN DE C(A)ESIOLA}

Se trata de una placa de mármol blanco de grano muy brillante que muestra una pátina amarillenta y que se encuentra partida en tres trozos que casan sin dificultad. Esta fractura, no obstante, no dificulta la lectura del texto, como tampoco lo hacen los pequeños fragmentos que ha perdido en su parte superior y en la esquina inferior derecha. La pieza tiene las medidas siguientes: $30 \times 30,3 \times 4 \mathrm{~cm}$, con la cara frontal apomazada. Presenta un marco moldurado en cyma reversa que hace que el campo epigráfico disponible tenga unas dimensiones de $20,5 \times 23 \mathrm{~cm}$. Se aprecian las finas líneas de guía del pautado horizontal, que no fueron borradas en el proceso final de ejecución de la pieza como era lo usual. La escritura es una capital cuadrada realizada mediante grabado profundo y refuerzos marcados, y en la que se aprecian algunos rasgos librarios en caracteres como $\mathrm{S}$ o $\mathrm{M}$. El módulo de las letras no es uniforme, oscilando entre 2,6-3,3 $\mathrm{cm}$ en 1 . 1, 2,3-3,3 cm en $1.2,2-3,3 \mathrm{~cm}$ en $1.3,2,3-4$ cm en 1.4 y 2,6-3,6 en 1. 5, a excepción de la primera I de 1.5 , de menores dimensiones $(1,6 \mathrm{~cm})$. La ordinatio es bastante descuidada, por no decir pésima, como se aprecia en la falta de uniformidad del módulo de las letras, en el amontonamiento de caracteres de

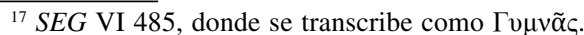

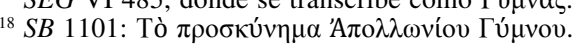

${ }_{19}$ Para los nombre griegos en la Península Ibérica, vide Lozano 1998.

${ }^{20}$ CIL VI 6460, 14229, 20770, 22455, 27587, 33583, 34695, 5688 (= CIL X $7135=A E 1990,22$, corrección de la lectura original Gymnas); cf. Solin 1996: 542; Solin 2003: 1250.
} 
la última línea, donde incluso llegan a trasgredir los límites del campo epigráfico, o en el vacat existente en 1. 2, que obliga al nombre de la difunta a salirse del campo epigráfico por la derecha; además, en 1.5 el cuadratario ha tenido que insertar forzadamente la I de Matri, acomodándola entre el lóbulo y el trazo diagonal de la $\mathrm{R}$, e incluso ha olvidado grabar dos de las letras de la fórmula funeraria final. Hay interpunciones triangulares con el vértice hacia abajo en 1l. 1, 2, 4 y 5, y quizá también en $1.3^{21}$, y dos hederae en forma de corazón en 1. 1, bastante toscas y sobredimensionadas. Particularidades paleográficas: M de arcos muy abiertos, con tres apoyos en la línea de caja; P y R con óculo cerrado; N con trazos verticales que tienden a inclinarse a la derecha; $\mathrm{E}$ y $\mathrm{F}$ con trazos horizontales rectos; travesaño de la $\mathrm{T}$ con cierta ondulación; $\mathrm{O}$ de módulo inferior al resto de caracteres. Se ignora la procedencia de esta pieza, que está depositada en la colección particular de D. Francisco Alcaide Juano ${ }^{22}$ (Fig. 2).
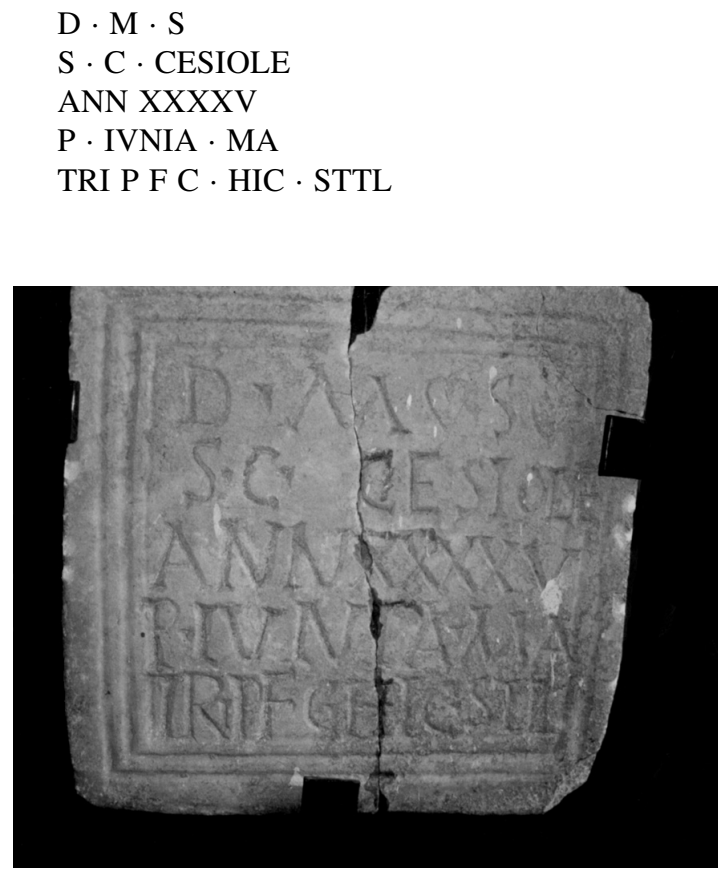

Figura. 2. Inscripción de Caesiola (Foto: S. Ordóñez Agulla).

\footnotetext{
${ }^{21}$ El trazo oblicuo izquierdo de esta posible interpunción parece vislumbrarse sobre el margen izquierdo del desconchón.

${ }^{22}$ Hemos de agradecer a D. Francisco Alcaide Juano el permiso para acceder a esta inscripción, agradecimiento que se ha de hacerse extensivo a D. Manuel Alonso por sus gestiones así como a R. Carande y J. C. Saquete por haber atendido amablemente nuestras consultas. Sobre otras piezas de esta colección, vide Sáez et alii 2003.
}

Como se puede observar son numerosas las anomalías que se acumulan en esta inscripción. Estas incluyen tanto la forma de presentar la onomástica de los dos personajes -el de la difunta, con praenomen, nomen abreviado y cognomen, el de la dedicante también con abreviatura del praenomen, o quizás del nomen-, como la anormalidad en la fórmula funeraria final, con el desarrollo de hic pero sin que se haya completado el formulario con $s($ ita $) e(s t)$. Entre los rasgos paleográficos que mueven a la sospecha destacan especialmente algunos caracteres de la 1.4 , como la R, el nexo TR, nada habitual, o incluso I, que no parecen de factura antigua; también el uso arbitrario de interpunciones y hederae es llamativo. La acumulación de anomalías debe llevar a considerar la posibilidad de encontrarnos ante una falsificación. A ello se suma el hecho de la falta de datos constatables sobre su origen y el encontrarse depositada en una colección particular. A este respecto, no se ha podido recabar dato alguno de su procedencia ni de las circunstancias de su hallazgo, tratándose de una pieza, según su propietario, procedente del mercado de antigüedades. La presencia conjunta de la dedicación a los Manes junto con el nombre del difunto en dativo y la fórmula $f$ (aciendum) c(uravit) es algo muy infrecuente en el territorio bético, pero no en epígrafes de Lusitania ${ }^{23}$, lo que quizá pudiera sugerir una posible proveniencia de la pieza del entorno lusitano o betúrico, que en cualquier caso debe quedar a título de hipótesis. En fin, teniendo presente las dudas y sospechas que suscita el texto en cuanto a su autenticidad y la posibilidad cierta de encontrarnos ante una falsificación, lo que no sería nada extraño habida cuenta de la existencia en Andalucía de mistificaciones de este tipo generadas por el intenso tráfico del mercado de antigüedades y los intereses de los coleccionistas ${ }^{24}$, procedemos a continuación a realizar el comentario de la pieza.

Sin duda la particularidad más señalada de esta inscripción en el ámbito onomástico es la consignación de $\mathrm{S}$ y $\mathrm{C}$ en 1. 2. Como se puede apreciar en la fotografía, es inequívoca la existencia de una interpunción tras este carácter. En esa formulación esperaríamos que se tratara del praenomen de la difunta, un uso epigráfico

\footnotetext{
${ }^{23}$ Una búsqueda mediante EDCS muestra que son contados los casos béticos en los que se constata esa combinación -CIL II 989; $A E$ 1995, 829-, frente a los documentados en Lusitania, hasta 65; en el caso concreto de la fórmula f(aciendum) $c$ (uravit) esta se emplea en la Bética en 109 ocasiones, de ellas 69 en zonas colindantes con Lusitania, mientras que en esta provincia la cifra asciende a 710 registros.

${ }^{24}$ Un argumento añadido a la posible falsedad de este epitafio está en las sospechas que han suscitado otras piezas de esta misma colección; vide los comentarios de A. Canto en $H E p$ 9, $517=A E 2003,920$ (ladrillo de los Argentarii) y HEp 13, $587=A E$ 2003, 900 (urna de Valeria Rustica).
} 
que, aunque no muy usual, sí está bien documentado en diferentes lugares del Imperio. El fundamental estudio de M. Kajava (Kajava 1994) ha puesto de relieve la importancia de este fenómeno, especialmente entre las mujeres de las clases altas, así como su uso, más esporádico, en otros niveles de la jerarquía social; también se hacía eco este investigador de la escasa presencia de este fenómeno en Hispania ${ }^{25}$. Como decíamos, en nuestra inscripción es incuestionable en 1. 2 la $\mathrm{S}$ en el lugar que se espera para un praenomen. En el repertorio del investigador finlandés figuran varios praenomina empleados por mujeres que empiezan por S: Salvia, Septuma, Servia, Sexta (Kajava 1994: 212213). Sin embargo en todos los ejemplos colacionados se expresan estos nombres con su abreviatura de tres letras -como es lo propio de los de género masculino (Salomies 1987: 49-50) ${ }^{26}$-, no de una sola. Solamente en una ocasión, $A E$ 1971, $244=I L N 1,155=A E 1982$, 677 (Forum Iulii), encontramos una formulación de un praenomen femenino con S: S (exta?) Satia Maxsuma, así desarrollado en el corpus de las inscripciones de Frejus y en sus editores precedentes, aunque el mismo Kajava afirmaba en su estudio que "the reading of the first S is uncertain". Es por ello que tenemos serias dudas sobre la identificación de ese primer caracter de 1. 1 de nuestra inscripción con un praenomen, y nos preguntamos, en este contexto de mala compaginación e interpunciones arbitrarias, si no estaríamos mejor ante un error del cuadratario al marcar la interpunción entre $\mathrm{S}$ y $\mathrm{C}$ cuando intentaba grabar un nomen que comenzara por ambas letras ${ }^{27}$. En ese sentido, pensamos que una posibilidad a barajar podrían ser los gentilicios Scantia, Scaniana, Scaeuia, Scandilia, o quizá mejor por su mayor difusión, Scribonia, todos ellos atestiguados en la Península Ibérica (Abascal 1994: 213), y lo suficientemente extensos como para que fuera necesario abreviarlos de manera que nomen y cognomen cupieran juntos en la misma línea, ahorrando espacio para un texto que, como se aprecia en el último renglón, tenía demasiada información para tan exiguo espacio en la placa ${ }^{28}$. En la epigrafía meridional se encuentra ocasionalmente algún ejemplo de un nombre abrevia-

\footnotetext{
${ }^{25}$ Los ejemplos hispanos son: $C I L$ II $666=H E p$ 4, $259=$ $A E$ 1991, 978, 945, 3372, 3680, 5307, 4592, 4623, 5933; HAE $1635=H E p 10,60=A E$ 2000, 692; RIT 570; HEp 4, 216; $H E p$ 4, 1000; $A E$ 1988, $806=A E$ 1994, $1015=H E p$ 6, 179; $A E$ 1954, 86 = AE 1972, 239; IRCP 71 .

${ }^{26}$ Vide $\mathrm{n}^{\mathrm{a}} 8$ para los escasos ejemplos masculinos de praenomen Sextus abreviado en $\mathrm{S}$.

${ }^{27}$ Descartamos la posibilidad de que se trate de la fórmula $s(u b) c(u r a)$, que no tiene sentido en esa posición, junto a un nombre de mujer en dativo y no en genitivo.

${ }^{28}$ ¿Quizá se trata de una placa procedente de un enterramiento colectivo en el que no sería necesario hacer constar, por obvio, el nomen de forma desarrollada?
}

do de forma similar, como la Ap(pia) Polla de CILA II.3, 939, el Cl(audius/a) Artem[----] de CILA II.2, 452 (Italica), o el también italicense Tib. Cl(audius) Festus de CILA II.2, 406.

En el caso de que optáramos por la presencia de un praenomen y un gentilicio ambos abreviados, tampoco se puede aventurar ningún desarrollo seguro de este último $^{29}$. Al ignorarse su procedencia no cabe contrastar la difusión de los posibles candidatos en los centros epigráficos que se manejaran; lo que sí puede pensarse es que se trataría de un nomen suficientemente conocido y familiar en el entorno de ubicación de la lápida como para no desarrollarlo por completo. Solo a título de ejemplo, y por su popularidad en la provincia, cabría pensar en una $C$ (aecilia), C(alpurnia), C(assia), C(laudia) o C(ornelia).

El cognomen de la difunta, en la forma en que se ha grabado en la inscripción, Cesiola, es desconocido en la epigrafía hispana. En la transcripción que de él realizamos, $C($ a)esiola, hasta la fecha solo se disponía de un único paralelo en el imperio, en la persona de Maelonia Caesiola, una de los tres libertos que en Mérida se encargaron de la conmemoración, con un altar ricamente decorado, de su patrono L. Maelonius Aper, un veterano de la legio VI Victrix vuelto a su ciudad de origen a mediados del siglo $\mathrm{II}^{30}$. Este $\operatorname{cog}$ nomen ha sido considerado de raíz indígena por los diversos autores que se han ocupado de él ${ }^{31}$, lo que viene refrendado por el propio nombre lusitano de este beneficiarius consularis, Maelonius, así como por el de sus conlibertos, Primitivos y Maelia. El desconocimiento de la procedencia de la pieza nos impide confirmar para esta ese carácter peregrino que se ha señalado para la emeritense, o considerar si no se trataría más bien de una derivación en diminutivo del muy extendido nombre latino Caesius, y como él, de esa raigambre ${ }^{32}$.

\footnotetext{
${ }^{29}$ La abreviación del nomen está bien documentada en la epigrafía meridional hispana; solo en la provincia de Sevilla se documenta en una quincena de casos, de ellos tres de gentilicios comenzando por C (CILA II.1, 56-57, Sevilla; CIL II.3, 850 , Carmona).

${ }^{30}$ CIL II $491=E E$ 8.2, p. 362 . Sobre este veterano, Roldán 1974: $n^{\circ}$ 536; Le Roux 1982: 223 n $^{\circ}$ 183; Schallmayer et alii 1990: 637-638 no 831; Nelis-Clément 2000: 85, 295.

${ }^{31}$ Untermann 1965: 82 Mapa 24: Caesius (como cognomen, casi exclusivo de la Hispania indoeuropea); García Iglesias 1973: 127: Caesiola, derivado de la raíz * ghaiso- 'punta, dardo', sobre la base de Albertos Firmat 1966: 70-71. También se considera un nombre peregrino en Ramírez Sádaba 2003: 63, 69, y Navarro y Ramírez Sádaba 2003: 126. Igualmente, figura entre los antropónimos indígenas de Lusitania en el estudio de Vallejo 2005: 244-245, 595-596, 732, entre las formas derivadas del radical indígena Caes-, en este caso en -olä; se añade además que la forma Ces(s)ius, - $a$, en algunos casos, es una variante de Caes-.

${ }^{32}$ En esa línea, Kajanto 1965: 167, sobre la base de $C I L$
} 
La dedicante aparece como $P$. Iunia. Aquí podríamos estar ante un praenomen abreviado, o bien ante la expresión de un nomen abreviado, cualquiera de los muchos que empiezan por $\mathrm{P}$, y la consignación de un nomen en función de cognomen ${ }^{33}$. Como tal cognomen, Iunia está bien atestiguado en un grupo de inscripciones hispanas ${ }^{34}$. No obstante, lo usual es que este nombre se encuentre empleado como gentilicio, siendo de hecho uno de los más frecuentes en Hispania, ocupando el noveno lugar en el repertorio de Abascal y con abundante representación en la epigrafía de la Bética, tanto en la lapidaria como en la anfórica (Abascal 1994: 163; Chic 1988: 211; Chic 2001: 481).

Con respecto a las fórmulas funerarias finales, la separación que impone la interpunción tras hic nos hace considerar más válido el desarrollo $s(i t) t(i b i)$ t(erra) l(evis) que la opción hic s(ita) e(st) [s(it) t(ibi)] t(erra) l(evis).

En función de lo dicho, la restitución que proponemos es la siguiente:

$D($ is) M(anibus) s(acrum) / SC(-ae) (vac.) C(a) esiol(a)e / ann(orum) XXXXV / P(---) Iunia ma/tri p(ientissimae? / iisimae?) f(aciendum) c(uravit) hic $<s($ ita $) e(s t)>s($ it $)$ t(ibi) t(erra) l(evis)

En lo que se refiere a la datación del texto, en principio esta habría de situarse en el siglo II d.C., más específicamente en su segunda mitad. Tanto la paleografía empleada, como el formulario, con la notación de la consagración a los Dioses Manes, que se incorpora al lenguaje epigráfico a partir del tránsito del siglo I al II d.C, y la abreviatura de la edad en ann., apuntan claramente a esa centuria (Stylow 1995: 222-223; Stylow 2002: 361). Otro elemento que apunta hacia esta datación es el empleo de las hederae, que aunque atestiguado en la Bética ya en época augustea, se generaliza en la epigrafía peninsular a partir de los flavios (Haley 1986: 130; Stylow 1995: 221 $\mathrm{n}^{\mathrm{a}}$ 21; Cebrián 2000: 97). Con todo, la abreviatura de los nomina, indicativa igualmente de una fecha avanzada (Haley 1991: 125; López y Stylow 1996:

II 491; Solin y Salomies 1994: 306; Abascal 1994: 44 y 309; Lörincz 1999: 20.

${ }^{33}$ Vid. Abascal 1994: 208 para una recopilación de ejemplos paralelos hispanos de este uso con $\mathrm{P}(---)$, como $P(---)$ Campana $\left(\right.$ CIL II $\left.{ }^{2} 14,1,212\right)$ o $P(---)$ Lauriola (RIT 632). Este uso se documenta desde inicios de la época imperial, vide Salomies 1987: $340 \mathrm{n}^{\mathrm{a}} 2$ y 357 .

${ }^{34}$ Aponia Iunia (CIL II 381 = Alarçao y Etienne 1976:56; CIL II 387 = Alarçao y Etienne 1976: 62), (---) Iunia (CIL II 568), Porcia Iunia (CIL II 3341 = CILA III.2, 605), Caecilia Iunia (CIL II $4311=$ RIT 439), Cornelia Iunia $(A E$ 1983, 613 = Fabre et alii 1982: 207 ss.).
296), posibilita prolongar la datación de la pieza hasta los primeros decenios del siglo III d.C. a juzgar por algunos testimonios béticos de este uso que cuentan con una asignación cronológica más precisa en época severiana $^{35}$.

\section{BIBLIOGRAFÍA}

Abascal Palazón, J. M. 1994: Los nombres personales en las inscripciones latinas de Hispania, Murcia.

Alarcão, J. y Etienne, R. 1976: Fouilles de Conimbriga II. Épigraphie et sculpture, Paris.

Alarcón Castellano, F. J. 1991: "Intervención arqueológica de emergencia Avd. de Sevilla $\mathrm{n}^{\circ}$ 2. Chipiona (Cádiz)”, Anuario Arqueológico de Andalucía 1991. III, 51-57.

Albertos Firmat, M. L. 1966: La onomástica personal primitiva de Hispania Tarraconense y Bética, Salamanca.

Alcázar Godoy, J., Suárez, A. y Alarcón, F. J. 1994: "Enterramientos infantiles en ánforas romanas. Estudio antropológico de un hallazgo excepcional”, Revista de Arqueología 164, 37-47.

Bonneville, J., Dardaine, S. y Le Roux, P. 1988: Belo 5. L'Épigraphie. Les inscriptions romaines de Baelo Claudia, Madrid.

Cebrián Fernández, R. 2000: Titulum fecit. La producción epigráfica en las tierras valencianas, Madrid.

Chic García, G. 1988: Epigrafía anfórica de la Bética II. Los rótulos pintados sobre ánforas olearias. Consideraciones sobre la annona, Sevilla.

Chic García, G. 2001: Datos para un estudio socioeconómico de la Bética. Marcas de alfar sobre ánforas olearias, Écija.

Fabre, G., Mayer, M. y Rodà, I. 1982: "Inscripciones alienae en museos y colecciones de la provincia de Barcelona", Ampurias 44, 185-240.

Ferrua, A. 1966: "Antiche iscrizioni inedite di Roma", Epigraphica 28, 18-43.

Fornell Muñoz, A. 2005: Las villae romanas en la Andalucía mediterránea y del estrecho, Jaén.

García Iglesias, L. 1973: Epigrafía Romana de Augusta Emerita, Madrid.

Gimeno Pascual, H. y Stylow, A. U. 2003: "Las inscripciones", J. Beltrán Fortes y J. R. López Rodríguez (coords.), El museo cordobés de Pedro Leonardo de Villaceballos, Málaga-Madrid, 149-218.

González, J. 1982: Inscripciones romanas de la provincia de Cádiz, Cádiz.

${ }^{35}$ CIL II $^{2} / 7,976=H E p ~ 4,182=A E$ 1991, 1027, de Regina. Otras piezas con nomina abreviados y datación a fines del siglo II o inicios del III son $C I L \mathrm{II}^{2} / 7,239,507,527,606$. 
Haley, E. W. 1986: Foreigners in Roman Imperial Spain. Investigations of geographical Mobility in the Spanish Provinces of the Roman Empire 30 B.C.- A.D. 284, Michigan.

Kajanto, I. 1965: The Latin cognomina, Helsinki.

Kajava, M. 1994: Roman female praenomina. Studies in the Nomenclature of Roman Women, Rome.

Lagóstena Barrios, L. 2001: La producción de salsas y conservas de pescado en la Hispania romana (siglo II a. C. - VI d. C.), Barcelona.

Le Roux, P. 1982: L'Armée romaine et l'organisation des provinces ibériques d'Auguste à l'invasion de 409, Paris.

López Palomo, L. A y Stylow, A. U. 1996: "Nuevo epígrafe de Herrera (Sevilla)", Anuario de Arqueología Cordobesa 7, 293-300.

Lörincz, B. 1999: Onomasticon Provinciarum Europae Latinarum, Wien.

Lozano Velilla, A. 1998: Die griechischen Personennamen auf der Iberischen Halbinsel, Heidelberg.

Muñoz García de Iturrospe, M. T. 1995: Tradición formular y literaria en los epitafios latinos de la Hispania cristiana, Vitoria.

Muñoz y Romero, T. 1858: Diccionario bibliográfico-histórico de los antiguos reinos, provincias, ciudades, villas, iglesias y santuarios de España, Madrid.

Navarro Caballero, M. y Ramírez Sádaba, J. L. 2003: Atlas antroponímico de la Lusitania romana, Mérida-Bordeaux.

Nelis-Clément, J. 2000: Les beneficiarii. Militaires et administrateurs au service de l'Empire (Ier siécle. a.C.-VIe s. p.C.), Bordeaux.

Pape, W. y Benseler, G. 1959: Wörterbuch der griechischen Eigennamen, Graz.

Ramírez Sádaba, J. L. 2003: "La integración social de los indígenas en la sociedad emeritense", Epigrafía y sociedad en Hispania durante el Alto Imperio: estructuras y relaciones sociales, Alcalá de Henares, 57-73.

Ramos Millán, A. 1981: "El alfar romano de 'El Olivar' (Chipiona, Cádiz). Aportación al estudio de las ánforas béticas de salazones", Gades 7, 5-25.

Ramos Millán, A. y Riesco García, J. C. 1983: "La villa romana de Las Canteras (Chipiona, Cádiz).
Procesos formativos y transformativos del registro arqueológico e inferencias preliminares del asentamiento", Cuadernos de Prehistoria y Arqueología de la Universidad de Granada 8, 375-416.

Ramos Millán, A., García Vargas, E., Osuna Vargas, M $^{\text {a }}$ M. y Cara Maldonado, S. 2001: "La carta de riesgo arqueológico de la alfarería romana de El Olivar de Chipiona (Cádiz). La actuación de urgencia de 2001 como gestión preventiva", Anuario Arqueológico de Andalucía 2001.III.1, 49-62.

Roldán Hervás, J. M. 1974: Hispania y el ejército romano. Contribución a la historia social de la España antigua, Salamanca.

Sáez Fernández, P., Ordóñez Agulla, S. y García-Dils de la Vega, S. 2003: "Inscripciones romanas inéditas en la provincia de Sevilla", Habis 34, 229-257.

Salomies, O. 1987: Die römischen Vornamen. Studien zur römischen Namengebung, Helsinki.

Schallmayer, E., Eibl, C., Ott, J., Preuss, G. y Wittkopf, E. 1990: Der römische Weihebezirk von Ostburken I: Corpus der griechischen und lateinischen Beneficiarier-Inschriften des Römischen Reiches, Stuttgart.

Solin, H. 1989: "Varia onomastica VIII. Gymnochares", Zeitschrift für Papyrologie und Epigraphik 77, 101-102.

Solin, H. 1996: Die Stadtrömischen Sklavennamen. Ein Namenbuch, II. Teil: griechische Namen, Stuttgart.

Solin, H. 2003: Die grieschischen Personnennamen in Rom. Ein Namenbuch, Berlin-New York.

Solin, H. y Salomies, O. 1994: Repertorium nominum gentilium et cognominum Latinorum, HildesheimZürich-New York.

Stylow, A. U. 1995: "Los inicios de la epigrafía latina en la Bética. El ejemplo de la epigrafía funeraria", F. Beltrán (ed.), Roma y el nacimiento de la cultura epigráfica en Occidente, Zaragoza, 219-238.

Stylow, A. U. 2002: "La epigrafía funeraria en la Bética", D. Vaquerizo (ed.), Espacios y usos funerarios en el Occidente romano, Córdoba, 353-368.

Untermann, J. 1965: Elementos de un atlas antroponímico de la Hispania antigua, Madrid.

Vallejo Ruiz, J. Ma 2005: Antroponimia indígena de la Lusitania romana, Vitoria.

Recibido: 21-03-2012

Aceptado: 26-06-2012 